\title{
Ultrastructure of the Nuclei and Intranuclear Microtubules of Paramecium aurelia
}

\author{
By A. JURAND AND G. G. SELMAN \\ Institute of Animal Genetics, West Mains Road, Edinburgh, EHg $3 \mathrm{JN}$
}

(Accepted for publication 13 November 1969)

\begin{abstract}
SUMMAR Y
Electron microscope observations on the micronucleus of Paramecium aurelia showed that the mitotic spindle was organized within the nuclear envelope. At the intranuclear metaphase plate stage some microtubules were attached to chromosomes by localized centromeres and other interzonal microtubules ran between the chromosomes. The interzonal microtubules elongated tenfold during telophase and were tightly packed in a narrow cylindrical membrane-limited stem of the spindle. Some microtubules persisted in the micronucleus during interphase and formed a layer near the nuclear envelope. There were scattered groups of microtubules in the isthmus of the macronucleus during its amitosis. The nuclear envelope remained intact. Large and small bodies persisted throughout the amitosis and the microtubules pass between them. Before the first postconjugal fission there was a stage during which the two macronuclear anlagen were separated by a sheet of cytoplasm only $0.3 \mu \mathrm{m}$. wide.
\end{abstract}

\section{INTRODUCTION}

In Paramecium and other ciliates there are two kinds of nuclei, macronuclei and micronuclei, which differ greatly in their size, amount of DNA, structure, function and mode of division at fission. In Paramecium aurelia between successive fissions there are normally two micronuclei, each about $3 \mu \mathrm{m}$. in diameter, which lie close to a single large ovoid macronucleus, which is about $35 \mu \mathrm{m}$. long and $\mathrm{I} 2 \mu \mathrm{m}$. across a minor diameter. It has been estimated that the macronucleus contains about 430 times as much DNA as the micronucleus (Woodard, Gelber \& Swift, I96ı; Woodard, Woodard, Gelber \& Swift, 1966). The macronucleus rather than the micronucleus must be concerned with growth and fission since Sonneborn (I938) showed that animals could survive for over a hundred fissions without a micronucleus but could not survive for more than one or two fission cycles without a macronucleus. Sonneborn (I946) also showed that the genes which determine the phenotype of the organism are located in the macronucleus. The micronuclei allow genetic recombination to take place at conjugation and at the first autogamy following conjugation; after autogamy and conjugation new macronuclei are derived from micronuclei.

Many light-microscopists have studied the behaviour of the nuclei of Paramecium aurelia during conjugation. Descriptions have been given by Hertwig (I 889), Maupas (1889), Sonneborn (1947, 1954), Kimball \& Gaither (1955), Jones (1956), Kościuszko (I965) and Jurand \& Selman (1969). Prometaphase and metaphase of the first meiotic division of the micronucleus presents the most favourable opportunity for observing 
and counting the chromosomes. These are small and numerous, however, and estimates of their diploid numbers in different stocks vary between 70 and 126 (Dippell, 1954; Jones, I956; Kościuszko, 1965). Paramecium aurelia has become popular for certain branches of genetical research (Beale, 1954; Preer, 1967), but cytological observations have not been able to assist these studies to any notable extent. It is possible that electron microscopy may ultimately be able to contribute as much useful information to the study of nuclear events in ciliates as it has already contributed to the study of their cytoplasmic organelles. The present paper includes electron microscope observations on the micronucleus and macronucleus of $P$. aurelia and describes the division of these two kinds of nuclei at fission. The organization of microtubules within the nuclei at division has not been described previously.

\section{METHODS}

Paramecium aurelia, mainly of stock 6o, was used and mass stocks were cultured by the method of Sonneborn (1950). A modified form of the fixative of Palade (1952) was prepared according to the formula quoted by Jurand \& Selman (I969). The addition of $0.03 \%$ calcium chloride to the osmic acid fixative was probably responsible for the preservation of the microtubules. The protozoa were first filtered through a cotton plug and then concentrated into an aqueous pellet by low speed centrifugation. The supernatant liquid was decanted and the fixative added at room temperature to the centrifuge tube which was shaken to disperse the protozoa. Fixation was for $30 \mathrm{~min}$. Dehydration was with graded ethanol + water mixtures and three changes of absolute ethanol (Io min. each). The protozoa were centrifuged down and the supernatant fluid decanted at each stage before fresh ethanol was added. Finally, the protozoa were passed through a $50+50$ mixture of I,2-epoxypropane + Araldite and embedded in Araldite by using a rotary shaker (Jurand \& Ireland, 1965). For light microscopy, sections were cut at I $\mu \mathrm{m}$. and stained with a $0.5 \%$ aqueous solution of toluidine blue made alkaline by adding $\mathrm{I} \%$ borax. For electron microscopy, sections were cut on a Porter-Blum Sorvall ultramicrotome, mounted on collodion-carbon coated grids and stained for $20 \mathrm{~min}$. with $\mathrm{r} \%$ potassium permanganate solution containing $2.5 \%$ uranyl acetate (Jurand \& Selman, 1969).

\section{OBSERVATIONS}

\section{The micronucleus and its mitotic stages}

When sectioned material stained with a basic dye is observed by light microscopy the interphase micronucleus of Paramecium aurelia appears as an achromatic sphere containing a central chromatic core. In thin sections observed by electron microscopy this dense core often appears hollow or cup-shaped and immediately surrounded by a thin moderately dense granular zone (Pl. I, fig. I). However, the bulk of the micronucleus contains material of low density outside the central core. In transverse section the two membranes of the nuclear envelope appear to join in some places over a distance of about $65 \mathrm{~nm}$. where there are probably nuclear pores. Immediately within the nuclear envelope there is a layer of microtubules (PI. I, fig. I). The microtubules run parallel to the adjacent nuclear envelope and in directions similar to those of lines of longitude on a globe. Thus they appear to diverge from a polar region of the 
nucleus and run round inside the nucleus to the opposite side. In earlier studies (Jurand, Beale \& Young, 1962) the type of fixative and embedding medium which were used did not allow these microtubules to be preserved. With the present techniques no interphase micronucleus has been observed which did not contain some microtubules.

When specimens of Paramecium aurelia are stained with methyl green and pyronin, the micronucleus appears green and does not stain with pyronin (A. Jurand, unpublished), indicating that the micronucleus contains little or no RNA. Therefore the dense central core of the interphase micronucleus probably consists of condensed chromatin. Similarly the micronuclei of $P$. bursaria and its suctorian parasite Podophyra parameciorum studied by Jurand \& Bomford (I965) were shown to contain little or no RNA. On the other hand Pasternak (1967) demonstrated autoradiographically that a macronucleate $P$. aurelia synthesized some RNA in the micronucleus.

In mitotic prophase the dense material near the centre of the micronucleus becomes irregularly dispersed (Pl. I, fig. 2). Microtubules are present near the nuclear envelope. In one case microtubules appeared to pass through the masses of prophase chromatin.

At metaphase many dense chromosomes are attached to parallel groups of microtubules at localized centromeres (Pl. I, fig. 3). Other interzonal microtubules are not attached to chromosomes but run between them. At the metaphase plate stage the microtubules of the spindle converge slightly toward the poles. However, the spherical nuclear envelope is everywhere intact and the metaphase plate of chromosomes and the spindle are organized entirely within the nuclear envelope. No centrioles have been observed.

At anaphase the interzonal microtubules and the nuclear envelope begin to elongate. The nuclear divisions of the two micronuclei within one protozoon are not always completely synchronous. In one serially sectioned paramecium, one micronucleus was in metaphase and its nuclear form was spherical, while the other was in anaphase, elongated in the direction of the spindle and had a length to breadth ratio of $\mathrm{I} \cdot 6: \mathrm{I}$.

During telophase the nucleus continues to elongate until the interzonal microtubules have increased to over Io times their initial length. The daughter sets of chromosomes are then separated by a long cylindrical tube bounded by the nuclear envelope which is still intact and contains densely packed interzonal microtubules (Pl. I, fig. 4). The micronuclear mitosis takes place in the last sixth of the interfission interval but it is completed before the fission furrow forms (Woodard et al. 196I).

\section{The macronucleus and its amitosis}

In thin-sectioned material, the mature macronucleus of Paramecium aurelia has been shown to contain two types of dense body (Sonneborn, I953; Jurand et al. 1962; Wolfe, 1967). The large type of body is usually between 0.5 and $\mathrm{I} \cdot 0 \mu \mathrm{m}$. in diameter and may contain a central region of low density between 0.2 and $0.5 \mu \mathrm{m}$. in diameter. The large dense bodies are pyronin-positive and were termed nucleoli by Nanney \& Rudzinska (1960). For each one of these large bodies there may be about a hundred dense small bodies 0.1 to $0.2 \mu \mathrm{m}$. in diameter. The small bodies, termed chromatin bodies by Nanney \& Rudzinska (1960), were shown to be Feulgen-positive by Wolfe (1967) who used a technique in which the macronuclei were first expanded by chelation of the divalent ions until the small bodies could be optically resolved. In electron micrographs of material stained with uranyl acetate alone, the small bodies appear to be much denser than the larger bodies (Pl. 3, fig. 8). The small bodies also have a 
more fibrillar ultrastructure than the large bodies which are more granular ( $\mathrm{Pl} .3$, fig. 9). Between the large and small bodies there is a low density of fibrillar material with fibril diameters of about $5 \mathrm{~nm}$. The macronucleus is bounded by a nuclear envelope which contains many nuclear pores with an internal diameter of $40 \mathrm{~nm}$. The morphology of the large and small bodies does not appear to change during the fission cycle.

Between fissions, the macronucleus does not normally appear to contain microtubules. In sectioned Paramecium aurelia, a group of microtubules has been observed within a non-dividing macronucleus in one instance only, but microtubules have been observed in a small proportion of macronuclei isolated by the method of Stevenson (I967). Vivier \& André (196I) observed disorganized microtubules in a macronucleus of $P$. caudatum.

In sections of Paramecium aurelia fixed when the fission furrow had formed, the macronucleus is shown constricted in the middle to form a shape like an hour glass (Pl. 2, fig. 5). At this stage the nuclear envelope is complete at all points and there is no evidence that it ever breaks down. The large and small bodies are present and appear to be ultrastructurally identical to the dense bodies in macronuclei at other stages of the fission cycle. In the isthmus, groups of microtubules run between the dense bodies and they are oriented in the direction of elongation of the nucleus (Pl. 2, fig. 6, 7). Fewer microtubules were observed in the main part of the macronucleus away from the isthmus. No attachment has been observed between the microtubules and the large or small bodies. When the fission furrow deepens to divide the protozoon, the macronucleus separates into two halves.

\section{A stage of close approach of the two macronuclear anlagen}

As a result of conjugation in Paramecium aurelia a synkaryon is formed in each conjugant. The two conjugants then normally separate and the synkaryon undergoes two mitoses to give four nuclei, two of which then enlarge and develop into macronuclear anlagen which are normally distributed one to each daughter animal at the first postzygotic fission so that each contains one new macronucleus. Jurand, Beale \& Young (1964) studied the ultrastructure of the macronuclear anlagen during their growth and development and described the origin of the large and small bodies. In the illustrated accounts of Hertwig (I889) and Maupas (I889) there is a stage at which the two macronuclear anlagen seem to press against each other. However, when the stages of conjugation and the nuclear events following conjugation were described and illustrated in the more recent account of Grell (1967) this pairing stage was ignored, which is not surprising because the temporary pairing and subsequent separation is of no known genetic or cytological significance.

The present observations confirm that, at a stage about midway between the separation of the exconjugants and the first postzygotic fission, the two macronuclear anlagen approach each other closely. At this stage the macronuclear anlagen are each about I I $\mu \mathrm{m}$. in diameter. By light microscopy, either no gap can be seen between the paired nuclei (Pl. 3, fig. Io) or the nuclei appear to be separated by a fine line. By electron microscopy the anlagen were observed to be separated by a layer of cytoplasm about $0.3 \mu \mathrm{m}$. wide (Pl. 3, fig. I I) and each nuclear envelope in this region was flattened over an area of about $13 \mu^{2}$. The macronuclear anlagen normally separate after this close approach, but Sonneborn (I947) and Nanney (1957) reported that under conditions 
of starvation the two anlagen in the exconjugants became irreversibly fused to form one macronucleus.

\section{DISCUSSION}

There are several points of similarity between the micronuclear mitosis in the holotrichous ciliate Paramecium aurelia and the heterotrichous ciliate Blepharisma studied by Jenkins (1967) and by Inaba \& Sotokawa (1968). In both cases the spindle is organized within the nuclear envelope in the absence of centrioles; the metaphase spindle includes microtubules which attach to chromosomes as well as interzonal microtubules which run between the two poles of the spindle and pass between the chromosomes. The remarkable elongation of the spindle which separates the daughter nuclei appears to take place by an active increase in length of the interzonal microtubules. In the present work, measurement of the external diameter of the microtubules gave results which did not differ significantly from a mean of $25 \mathrm{~nm}$. whatever the mitotic stage. This is in contrast to the finding of Hauser (1968) that in the suctorian Paracineta limbata the microtubules have a lesser diameter after the spindle elongates. In Paramecium, Blepharisma, Paracineta and the peritrichous ciliates Epistylis and Vorticella observed by Carasso \& Favard (1965), the nuclear envelope of the micronucleus is preserved intact during the entire mitotic division. For Blepharisma, Jenkins (1967) and Inaba \& Sotokawa (1968) observed that a new nuclear envelope formed round the daughter masses of chromatin within the old nuclear envelope at telophase. It is not known whether Paramecium is similar in this respect.

In this paper it is accepted that the large dense bodies ( 0.5 to I $\mu \mathrm{m}$. in diameter) within the macronucleus contain RNA and are nucleoli, while the small dense bodies ( 0.1 to $0.2 \mu \mathrm{m}$. in diameter) contain DNA and consist of condensed chromatin. This represents a re-interpretation of the results of Jurand et al. (I962). The observations made by these authors after staining with methyl green and pyronin, and after staining by the Feulgen method, and by ultraviolet microscopy following extraction with ribonuclease or deoxyribonuclease, now appear to support the present interpretation.

There is no evidence that the large or small bodies of the macronucleus change their morphology during the fission cycle of Paramecium aurelia. On the other hand in hypotrichous ciliates, both nucleolar material and condensed chromatin becomes temporarily dispersed in a reorganization band associated with the duplication of DNA (Gall, I959; Inaba \& Suganuma, I966; Kluss, I962). If the duplication of DNA in the macronucleus of $P$. aurelia took place asynchronously in different parts of the nucleus any associated morphological changes might be difficult to detect. In very young macronuclear anlagen there are no large or small bodies (Jurand et al. 1964), and the chromatin appears uniformly dispersed.

In the macronucleus of Paramecium aurelia, nucleolar material and condensed chromatin can be distinguished by their ultrastructure without reference to the size of the body in which these materials are aggregated, so that a small nucleolar fragment may be identified by its granular ultrastructure. There are similar fibrillar elements both within the small bodies of condensed chromatin and in the background material between them, so that the background material may contain diffuse chromatin. Wolfe (1967) reached similar conclusions by using a technique in which macronuclei were stretched on an air-water interface. There is no known relationship between the 
chromosomes observed within the micronuclei at metaphase and the small bodies found in the macronucleus, except that they both contain DNA.

Oriented bundles of microtubules were observed predominantly in the isthmus of macronuclei during amitosis and they were rarely found in macronuclei from protozoa not in fission, so there is circumstantial evidence that the microtubules play a role in the macronuclear division. However, since no attachment has been observed between the microtubules and any other structure within the macronucleus, there is no evidence that they help to segregate the genomic units. Tamura, Tsuruhara \& Watanabe (I 969) observed microtubules stretched along the isthmus of a dividing macronucleus of Tetrahymena pyriformis and Carasso \& Favard (1965) observed microtubules in the dividing macronucleus of the peritrichous ciliate Campanella umbellaria.

Sonneborn (1957) showed that the heterozygous and highly polyploid macronucleus of Paramecium aurelia retained its heterozygosity after hundreds of fissions and this observation supported his suggestion that the macronucleus is made up of diploid units which are distributed intact between the daughter macronuclei at fission (see Raikov, 1968). However, there are no ultrastructural observations which can be quoted in support of the existence of the diploid units. Amitosis has been regarded as a primitive and perhaps irregular form of nuclear division for which it is difficult to imagine an orderly duplication and equal distribution of daughter genomic units in the absence of any kind of mitosis. A similar conceptual difficulty exists when the duplication of the bacterial chromosome is considered, yet bacterial chromosomes appear to duplicate efficiently.

We wish to thank Professor G. H. Beale, F.R.S., for his interest and for his comments on a draft of this paper.

\section{REFERENCES}

Beale, G. H. (I954). The Genetics of Paramecium aurelia. Cambridge University Press.

Carasso, N. \& Favarid, P. (i965). Microtubules fusoriaux dans les micro- et macronucleus de ciliés péritriches en division. Journal de Microscopie 4, 395.

Dippell, R. V. (1954). A preliminary report on the chromosomal constitution of certain variety 4 races of Paramecium aurelia. Caryologia 6 (Suppl.), I 109.

GaLL, J.G. (I959). Macronuclear duplication in the ciliated protozoan Euplotes. Journal of Biophysical and Biochemical Cytology $\mathbf{5}, 295$.

Grell, K. G. (1967). Sexual reproduction in protozoa. Research in Protozoology. Ed. by T. Chen, vol. 2, p. I47. Oxford: Pergamon Press.

HAUSER, M. (1968). Die Bildung von Spindelfasern im Mikronucleus des Suktors Paracineta limbata. Zeitschrift für Naturforschung $\mathbf{2 3}$ b, 887.

Hertwig, R. ( I889). Über die Conjugation der Infusorien. Abhandlungen der Bayerischen Akademie der Wissenschaften 17, I5I.

INABA, F. \& SоTOKAWA, Y. (1968). Electron-microscopic observation on nuclear events during binary fission in Blepharisma wardsi (Ciliata, Heterotrichida). Japanese Journal of Genetics 43, 335.

INABA, F. \& SugANuma, Y. (I966). Electron microscopy of the nuclear apparatus of Urostyla grandis, a hypotrichous ciliate. Journal of Protozoology 13, 137.

JENkINs, R. A. (1967). Fine structure of division in ciliate protozoa. I. Micronuclear mitosis in Blepharisma. Journal of Cell Biology 34, 463.

JoNes, K. W. (1956). Nuclear differentiation in Paramecium. Ph.D. thesis, Aberystwyth University.

Jurand, A., Beale, G. H. \& Young, M. R. (I962). Studies on the macronucleus of Paramecium aurelia. I. (With a note on ultra-violet micrography.) Journal of Protozoology 9, 122.

Jurand, A., Beale, G. H. \& Young, M. R. (1964). Studies on the macronucleus of Paramecium aurelia. II. Development of macronuclear anlagen. Journal of Protozoology II, 49 I. 
JURAND, A. \& BOMFORD, R. (1965). The fine structure of the parasitic suctorian Podophyra parameciorum. Journal de Microscopie 4, 509.

Jurand, A. \& Ireland, M. J. (1965). A slow rotary shaker for embedding in viscous media. Stain Technology 40, 233.

Jurand, A. \& Selman, G. G. (1969). The Anatomy of Paramecium aurelia. London: Macmillan.

Kimball, R. F. \& Gaither, N. (I955). Behaviour of nuclei at conjugation in Paramecium aurelia. I. Effect of incomplete chromosome sets and competition between complete and incomplete nuclei. Genetics 40, 878 .

KLuss, B. C. (I962). Electron microscopy of the macronucleus of Euplotes eurystomus. Journal of Cell Biology 13, 462.

Kościuszko, H. (1965). Karyologic and genetic investigations in syngen I of Paramecium aurelia. Folia Biologica, Krakow r3, 339.

Maupas, E. ( I 889). La rajeunissement karyogamique chez les ciliés. Archives de Zoologie Expérimental et Générale 7, 149.

NANNEY, D. L. (1957). Mating type inheritance at conjugation in variety 4 of Paramecium aurelia. Journal of Protozoology 4, 89.

Nanney, D. L. \& Rudzinska, M. A. (1960). Protozoa. The Cell : Biochemistry, Physiology, Morphology, vol. 4, p. I09. Ed. by J. Brachet and A. E. Mirsky. New York: Academic Press.

Palade, G. E. (1952). A study of fixation for electron microscopy. Journal of Experimental Medicine 95, 285 .

PASTERnAK, J. (1967). Differential genic activity in Paramecium aurelia. Journal of Experimental Zoology $\mathbf{1 6 5}, 395$.

Preer, J. R. (1967). Genetics of the Protozoa. In Research in Protozoology, vol. 3, p. 129. Ed. by T. Chen. Oxford: Pergamon Press.

RaIkov, I. B. (1968). The macronucleus of ciliates. In Research in Protozoology, vol. 3, p. I. Ed. by T. Chen. Oxford: Pergamon Press.

SONNEBorn, T. M. (1938). Sex behaviour, sex determination, and the inheritance of sex in fission and conjugation in Paramecium aurelia. Genetics 23, 168.

SONNEBORN, T. M. (1946). Inert nuclei: inactivity of micronuclear genes in variety 4 of Paramecium aurelia. Genetics 3I, $23 \mathrm{I}$.

Sonneborn, T. M. (1947). Recent advances in the genetics of Paramecium and Euplotes. Advances in Genetics I, 263.

SONNEBORN, T. M. (1950). Methods in the general biology and genetics of Paramecium aurelia. Journal of Experimental Zoology $\mathbf{1 3}, 87$.

SONNEBorn, T. M. (I953). Electron micrographs of ultra thin sections of the nuclei of Paramecium aurelia. Microbial Genetics Bulletin 7, 24.

SONNEBoRN, T. M. (1954). Patterns of nucleocytoplasmic integration in Paramecium. Caryologia 6 (Suppl.), 307.

SONNEBORN, T. M. (I 957). Breeding systems, reproductive methods and species problems in Protozoa. In The Species Problem, 50, I 55. Ed. by E. Mayr. American Association Adv. Sci. Publ.

Stevenson, I. (1967). A method for the isolation of macronuclei from Paramecium aurelia. Journal of Protozoology 14, 412.

Tamura, S., Tsuruhara, T. \& Watanabe, Y. (1969). Function of nuclear microtubules in macronuclear division of Tetrahymena pyriformis. Experimental Cell Research 55, 35I.

VIVIER, E. \& ANDRÉ, J. (I96I). Existence d'inclusions d'ultrastructure fibrillaire dans le macronucleus de certaines souches de Paramecium caudatum Ehr. Comptes Rendu Hebdomadaire des Séances de l'Academie des Sciences 252, 1848.

Wolfe, J. (1967). Structural aspects of amitosis: a light and electron microscope study of the isolated macronuclei of Paramecium aurelia and Tetrahymena pyriformis. Chromosoma 23, 59 .

Woodard, J., Gelber, B. \& SwIFT, H. (196I). Nucleoprotein changes during the mitotic cycle in Paramecium aurelia. Experimental Cell Research 23, 258.

Woodard, J., Woodard, M., Gelber, B. \& Swift, H. (i966). Cytochemical studies of conjugation in Paramecium aurelia. Experimental Cell Research 4I, 55. 


\section{EXPLANATION OF PLATES}

Scale marks correspond to I $\mu \mathrm{m}$. unless otherwise indicated.

Plate I

Fig. I. The interphase micronucleus has a central core of dense material surrounded by a thin granular layer of intermediate density. The low-density region of the nucleus near the nuclear envelope contains linear elements which are microtubules, most of which are seen here in longitudinal section. The nuclear envelope has inner and outer membranes. The dense material which occurs at intervals in the nuclear envelope represents the position of nuclear pores. Electron micrograph. $\times 28,000$.

Fig. 2. Part of a section through a micronucleus in prophase. The dense material (chromatin, $c h$ ) is dispersed in irregular clumps. Several bundles of microtubules are seen in transverse section and the microtubules all appear as circular profiles. Electron micrograph. $\times 37,000$.

Fig. 3. The dense chromosomes are arranged in a metaphase plate on an intranuclear spindle. The spindle is viewed laterally and some microtubules appear to terminate at localized regions of the chromosomes, while others run between the chromosomes. The arrowed chromosome has a centromere where microtubules are attached and a distal arm in the plane of the section. At the poles of the spindle, microtubules terminate at the nuclear envelope which remains intact. Electron micrograph. $\times$ I 8,000 .

Fig. 4. A detail of the telophase spindle remnant is shown in the long and narrow region between the daughter nuclei. The cylindrical bundle of tightly packed microtubules has been sectioned longitudinally and is bounded by the nuclear envelope. Electron micrograph. $\times 37,000$.

\section{Plate 2}

Fig. 5. A longitudinal section through a paramecium in fission shows the macronucleus in amitosis. It is elongated and constricted in the middle to form an isthmus at the level of the fission furrow $(f)$. Light micrograph of $\mathrm{I} \mu \mathrm{m}$. Araldite section stained with toluidine blue. $\times 88 \mathrm{o}$.

Fig. 6. A longitudinal section through part of a macronucleus during amitotic division, to show the region of the isthmus at the level of the fission furrow $(f)$. The constricted macronucleus is bounded by the nuclear envelope and large and small dense bodies can be seen. The arrows indicate the position of groups of microtubules. Electron micrograph. $\times$ I I, 000.

Fig. 7. Part of the field of Fig. 6 at higher magnification, showing a few of the microtubules $(m)$ which run in the matrix between the large and small bodies in the direction of elongation of the macronucleus. The double nuclear envelope $(n)$ is seen clearly wherever it is perpendicular to the plane of the section. Electron micrograph. $\times 38,000$.

\section{Plate 3}

Fig. 8. Macronucleus from a section stained only with $2.5 \%$ uranyl acetate solution after osmic fixation. The small bodies appear darker because of their greater affinity for uranyl acetate. Electron micrograph. $\times 16,000$.

Fig. 9. A detail from a macronucleus showing that the small bodies $(s)$ have a fine irregular fibrillar ultrastructure while, between the bodies, there is a matrix of similar fibrillar material of much lower density. The large body $(l)$ has a more granular ultrastructure and $20 \mathrm{~nm}$. granules are more distinct near the edges of the large body, while its interior is more fibrillar. Electron micrograph. $\times 60,000$.

Fig. 10. The two macronuclear anlagen (arrowed) are shown at the pairing stage which occurs between the beginning of conjugation and the first postconjugal fission. Light micrograph of $\mathrm{I} \mathrm{nm}$. Araldite section stained with toluidine blue. $\times$ I 200 .

Fig. II. Electron micrograph showing two macronuclear anlagen at the pairing stage. The layer of cytoplasm separating the two anlagen is about $0.3 \mu \mathrm{m}$. The anlagen at this stage contain only a few scattered dense bodies. $\times 6000$. 
Journal of General Microbiology, Vol. 6o, No. 3

Plate I
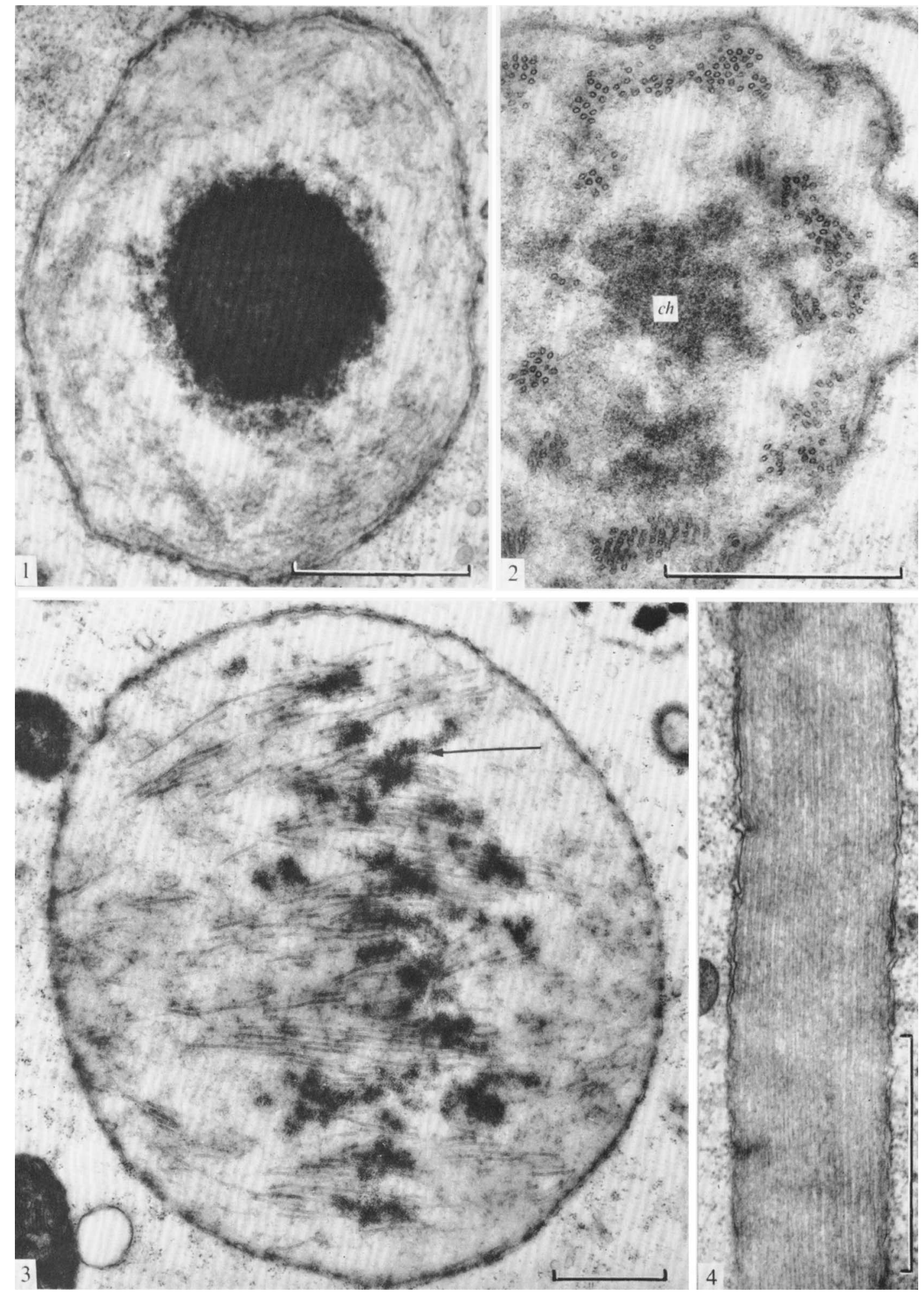

A. JURAND ANI G. G. SELMAN

(Facing p. 364) 
Journal of General Microbiology, Vol. 60, No. 3

Plate 2
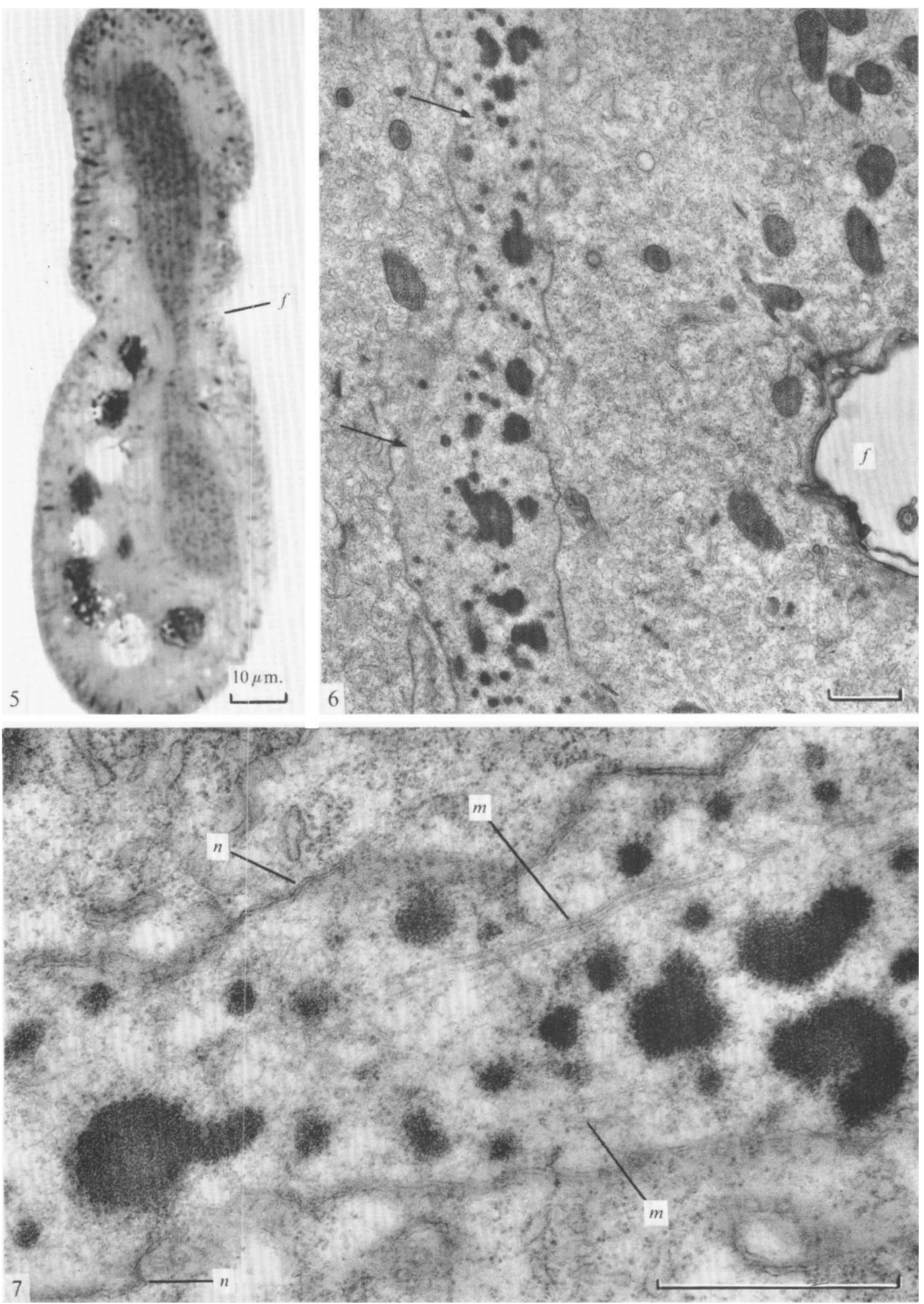

A. JURAND AND G. G. SELMAN 
Journal of General Microbiology, Vol. 60, No. 3

Plate 3
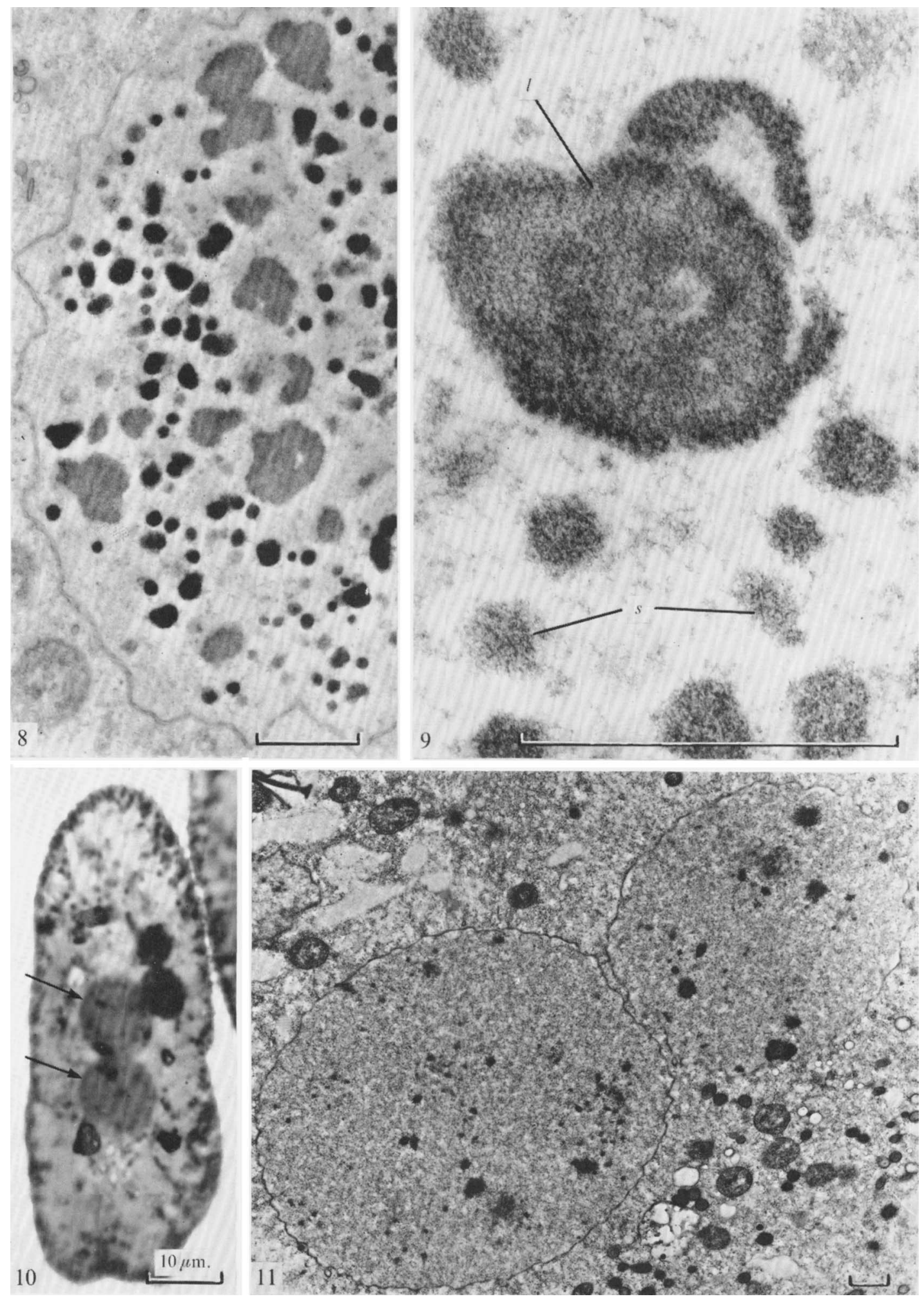

A. JURAND AND G. G. SELMAN 